\title{
COVID-19 and Genetic Variants of Protein Involved in the SARS-CoV-2 Entry into the Host Cells
}

\author{
Andrea Latini ${ }^{1}$, Emanuele Agolini ${ }^{2}{ }^{-1}$, Antonio Novelli ${ }^{2}{ }^{-1}$, Paola Borgiani ${ }^{1}$, \\ Rosalinda Giannini ${ }^{3}$, Paolo Gravina ${ }^{3}$, Andrea Smarrazzo ${ }^{4}$, Mario Dauri ${ }^{5}$, \\ Massimo Andreoni 6,7, Paola Rogliani ${ }^{8}$, Sergio Bernardini ${ }^{9}$, Manuela Helmer-Citterich ${ }^{10}$, \\ Michela Biancolella ${ }^{3,10}$ and Giuseppe Novelli $1,3,11,12, *$ (D) \\ 1 Department of Biomedicine and Prevention, Tor Vergata University Hospital, 00133 Rome, Italy; \\ latini.andrea@hotmail.com (A.L.); borgiani@uniroma2.it (P.B.) \\ 2 Laboratory of Medical Genetics, Bambino Gesù Children's Hospital, IRCCS, 00165 Rome, Italy; \\ emanuele.agolini@opbg.net (E.A.); antonio.novelli@opbg.net (A.N.) \\ 3 Medical Genetics Laboratory, Tor Vergata University Hospital, Rome, 00133 Rome, Italy; \\ rosalinda.giannini21@gmail.com (R.G.); paolo.gravina@ptvonline.it (P.G.); \\ michelabiancolella@gmail.com (M.B.) \\ 4 UOC Pediatria, Bambino Gesù Children's Hospital, IRCCS, 00165 Rome, Italy; andrea.smarrazzo@opbg.net \\ 5 Department of Clinical Sciences and Translational Medicine, Tor Vergata University Hospital, 00133 Rome, \\ Italy; dauri@med.uniroma2.it \\ 6 Department of Systems Medicine, Tor Vergata University Hospital, 00133 Rome, Italy; andreoni@uniroma2.it \\ 7 Infectious Diseases Clinic, Tor Vergata University Hospital, 00133 Rome, Italy \\ 8 Unit of Respiratory Medicine, Department of Experimental Medicine, Tor Vergata University Hospital, \\ 00133 Rome, Italy; rogliani@uniroma2.it \\ 9 Department of Experimental Medicine and Biochemical Sciences, Tor Vergata University Hospital, \\ 00133 Rome, Italy; bernardini@med.uniroma2.it \\ 10 Department of Biology, Tor Vergata University Hospital, 00133 Rome, Italy; citterich@uniroma2.it \\ 11 IRCCS Neuromed, 86077 Pozzilli, Italy \\ 12 Department of Pharmacology, School of Medicine, University of Nevada, Reno, NV 89557, USA \\ * Correspondence: novelli@med.uniroma2.it; Tel.: +39-06-20900668
}

Received: 13 July 2020; Accepted: 26 August 2020; Published: 27 August 2020

check for updates

\begin{abstract}
The recent global COVID-19 public health emergency is caused by SARS-CoV-2 infections and can manifest extremely variable clinical symptoms. Host human genetic variability could influence susceptibility and response to infection. It is known that ACE2 acts as a receptor for this pathogen, but the viral entry into the target cell also depends on other proteins. The aim of this study was to investigate the variability of genes coding for these proteins involved in the SARS-CoV-2 entry into the cells. We analyzed 131 COVID-19 patients by exome sequencing and examined the genetic variants of TMPRSS2, PCSK3, DPP4, and BSG genes. In total we identified seventeen variants. In PCSK3 gene, we observed a missense variant (c.893G >A) statistically more frequent compared to the EUR GnomAD reference population and a missense mutation (c.1906A>G) not found in the GnomAD database. In TMPRSS2 gene, we observed a significant difference in the frequency of c.331G $>A$, c. $23 \mathrm{G}>\mathrm{T}$, and c.589G $>$ A variant alleles in COVID-19 patients, compared to the corresponding allelic frequency in GnomAD. Genetic variants in these genes could influence the entry of the SARS-CoV-2. These data also support the hypothesis that host genetic variability may contribute to the variability in infection susceptibility and severity.
\end{abstract}

Keywords: COVID-19; SARS-CoV-2; genetic variants; host genetic variability; TMPRSS2; PCSK3 


\section{Introduction}

The ongoing pandemic of coronavirus disease 2019 (COVID-19) certainly represents one of the most important clinical emergencies of the 21st century [1]. The pathogen responsible for this global epidemic is the novel beta-coronavirus, known as SARS-CoV-2. It belongs to the family of Coronaviruses $(\mathrm{CoV})$, a large family of viruses that includes Middle East Respiratory Syndrome (MERS-CoV) and Severe Acute Respiratory Syndrome (SARS-CoV) [2]. COVID-19 disease can manifest extremely variable clinical symptoms, ranging from very mild sub-clinical symptoms to Acute Respiratory Distress Syndrome (ARDS). These highly heterogeneous phenotypes also seem to depend on patient age, gender, and underlying health conditions [3]. Furthermore, this phenotypic variability could be explained by inter-individual genetic unevenness. In particular, host susceptibility and response to infection, could be influenced also by genetic variants in genes coding for proteins with an active role in the entry and spread of SARS-CoV-2.

It is known that SARS-CoV-2 employs its spike type I transmembrane glycoprotein (S protein) to bind the host surface protein ACE2 (Angiotensin-2 Conversion Enzyme) and mediate cell entry [4]. However, recent studies have shown that the entry of SARS-CoV-2 into the target cell also depends on other proteins [5]. Indeed, entry requires $S$ protein priming by cellular proteases, which cleave $S$ protein in S1 and S2 subunits and allows fusion of viral and cellular membranes, mediated by S2. In particular, SARS-CoV-2 uses host serine protease TMPRSS2 for S protein priming and for entry into primary target cells [6].

The high spreading capacity of the infection suggests that there are other mechanisms which actively contribute to viral entry into the cell [5]. In particular, the $S$ protein contains a proprotein convertase (PPC) motif, a functional furin-cleavage sequence (RRAR) located at the junction between the S1 and S2 subunits [7]. Furin (PCSK3) is an ubiquitous membrane-bound protease highly expressed in the lungs. High levels of furin protease in the human respiratory tract may activate the SARS-CoV-2 $\mathrm{S}$ protein, cleaving it into S1 and S2 subunits. Hence, furin pre-activation could allow SARS-CoV-2 to be less dependent on target cells, enhancing its entry into target cells with relatively low expression of TMPRSS2, in a mechanism similar to that observed in avian influenza H5N1 virus [8].

Molecular bioinformatic studies have identified other possible host-receptor interactions in the entry of SARS-CoV-2 that facilitate the efficiency of viral particles spread egress. These include the dipeptidyl peptidase 4 (DPP4) protein or CD26, used as cell entry receptor by the MERS-CoV [9], and CD147, also known as Basigin (BSG), a transmembrane glycoprotein belonging to the immunoglobulin superfamily which acts as receptor of host cells for SARS-CoV-2 invasion [10,11].

In a previous paper we investigated the genetic variants of ACE2 gene [12] in a cohort of 99 Italian SARS-CoV-2-positive patients by direct exome sequencing, and we found no relevant association between the observed variants and COVID-19 disease [13]. The aim of the present study was to analyze, in an Italian cohort of 131 patients (99 studied in the previous paper for ACE2 and 32 newly recruited), the coding region and the exon/intron junction of the other four genes (TMPRSS2, PCSK3, DPP4, BSG) [12] involved in the SARS-CoV-2 entry into target cells.

\section{Materials and Methods}

\subsection{COVID-19 Patients}

We enrolled 131 COVID-19 patients hospitalized at the University Hospital of Rome "Tor Vergata" and Bambino Gesù Children's Hospital in Rome, during the period between March and May 2020. All patients were diagnosed with COVID-19 based on clinical evidence and confirmed by viral RNA detection at oropharyngeal and nasopharyngeal swabs by real-time PCR. The patients were clustered in three groups: (1) severe, according to respiratory impairment, requiring non- invasive ventilation; (2) extremely severe, defined as respiratory failure, requiring invasive ventilation and intensive care unit admission, (3) asymptomatic, according to absence of clinical symptoms and not requiring hospitalization or ventilation. 
The majority of the enrolled patients were males (82 males, 49 females). Median age was 63.7 years (range: 2-92 years), seventy-eight patients were under 65 years old. Ten patients were children (median age was 11.5 years) showing a severe form of the disease but none of them had Kawasaki-like syndrome [12,14]. Peripheral blood samples were collected. The analytical procedure received approval by the local ethics committee at the University Hospital of Rome Tor Vergata (protocol no. 50/20). The study was conducted in agreement with the principles of the Declaration of Helsinki. Informed written consent was obtained from each patient.

\subsection{Whole Exome Sequencing and Data Preprocessing}

Library preparation and whole exome capture were performed by using the Twist Human Core Exome Kit (Twist Bioscience, South San Francisco, CA, USA) according to the manufacture's protocol and sequenced on the Illumina NovaSeq 6000 platform. The BaseSpace pipeline (Illumina, Inc., San Diego, CA, USA) and the TGex software (LifeMap Sciences, Inc., Alameda, CA, USA) were used for the variant calling and annotating variants, respectively. Sequencing data were aligned to the hg19 human reference genome. Based on the guidelines of the American College of Medical Genetics and Genomics, a minimum depth coverage of $30 \times$ was considered suitable for analysis. Variants were examined for coverage and Qscore (minimum threshold of 30) and visualized by the Integrative Genome Viewer (IGV).

\subsection{Statistical Analysis}

Differences in alleles frequencies between groups were evaluated by the Pearson $\chi^{2}$ test or by Fisher's exact test, as requested according to the numbers of samples in the compared groups. $p$-values less than 0.05 were considered statistically significant. The Hardy-Weinberg equilibrium was evaluated, where possible, by the Pearson $\chi^{2}$ test.

\section{Results}

In total we identified in the four investigated genes, 17 genetic variants. The observed variants and their frequency distribution are reported in Table 1.

The variants frequencies detected in our examined cohort for the BSG and DPP4 genes were not statistically different with respect to the frequencies listed in the GnomAD database [15] for the European non-Finnish population [16].

In PCSK3 gene, we identified five different germline variants, one intronic (c.372+5G $>\mathrm{A}$ ) and four missense (c.128C>T, p.Ala43Val; c.436G >A, p.Gly146Ser; c.893G>A, p.Arg298Gln; c.1906A>G, p.Ile636Val). The allelic frequency of these variants in GnomAD for the EUR reference population is very low and we detected a statistically significant difference for the c.893G $>$ A, (p.Arg298Gln) compared to our cohort $(p=0.0047)$. Instead, the variant c.1906A $>G$, (p.Ile636Val) identified in a heterozygous male, is not present in the GnomAD database. (Table 1). The missense variants mapping on the protein known structure (G146S and R298Q) are both at more than $20 \AA$ from the protein active site.

In TMPRSS2 gene, we identified five different missense variants (c.22G>C, p.Gly8Arg; c.23G $>\mathrm{T}$, p.Gly8Val; c.193G >A, p.Ala65Thr; c.331G >A, p.Gly111Arg; c.589G >A, p.Val197Met). The last variant is the only one mapping on the catalytic domain, far from the active site $(>30 \AA)$. The missense variant c.193G > A, (p.Ala65Thr), and c.589G >A (p.Val197Met) were found in COVID-19 patients with frequencies in line with those reported for the reference population in the GnomAD database. Instead, a significant difference was detected for the c.331G $>A$, (p.Gly111Arg), a very rare allele in the European non-Finnish population of the GnomAD database, observed in our cohort in a heterozygous male patient $(p=0.0163)$. Furthermore, we observed a lower frequency of $c .23 \mathrm{G}>\mathrm{T}$ (p.Gly8Val) variant allele and of c.22G>C, p.Gly8Arg in COVID-19 patients, compared to allelic frequency of these variants in GnomAD for the EUR reference population ( $p=0.0446$ and $p=0.0228$, respectively). 
Table 1. Comparison of allelic counts (variant vs. wild type alleles) between our Italian population of SARS-CoV2 Positive Patients and Europeans (GnomAD database).

\begin{tabular}{|c|c|c|c|c|c|c|c|c|c|c|c|c|c|}
\hline Gene & Nr. & dbSNP & $\begin{array}{c}\text { Position } \\
\text { (Hg19) }\end{array}$ & Coding & Protein & Genotype (n) & Variant Type & $\begin{array}{l}\text { Allelic } \\
\text { Count }\end{array}$ & $\begin{array}{c}\text { Allelic Count } \\
\text { EUR (GnomAD) }\end{array}$ & $\begin{array}{c}\text { Allelic } \\
\text { Frequency }\end{array}$ & $\begin{array}{l}\text { Allelic Frequency } \\
\text { EUR (GnomAD) }\end{array}$ & $p$-Value & H.w. \\
\hline \multirow{4}{*}{$B S G$} & 1 & rs201850688 & $\begin{array}{l}\text { Chr19: } \\
572671\end{array}$ & c. $37 \mathrm{C}>\mathrm{G}$ & p.Leu13Val & Het (1) & Missense_variant & 1 vs. 261 & 87 vs. 66377 & $1 / 262=0.004$ & $17240 / 86164=0.001$ & $p=0.2928$ & $\begin{array}{l}\text { Not possible to } \\
\text { calculate }\end{array}$ \\
\hline & 2 & rs11551906 & $\begin{array}{l}\text { Chr19: } \\
572680\end{array}$ & c. $46 \mathrm{~A}>\mathrm{G}$ & p.Thr16Ala & Het (2) & Missense_variant & 2 vs. 260 & 1384 vs. 62996 & $2 / 262=0.008$ & $1384 / 64380=0.021$ & $p=0.1919$ & In equilibrium \\
\hline & 3 & rs144824657 & $\begin{array}{l}\text { Chr19: } \\
577782\end{array}$ & c.76G $>\mathrm{T}$ & p.Val26Phe & Het (1) & Missense_variant & 1 vs. 261 & 414 vs. 4028078 & $1 / 262=0.004$ & $414 / 40322=0.01$ & $p=0.5306$ & $\begin{array}{l}\text { Not possible to } \\
\text { calculate }\end{array}$ \\
\hline & 4 & rs41276870 & $\begin{array}{l}\text { Chr19: } \\
\text { 579501 }\end{array}$ & c. $417 \mathrm{C}>\mathrm{T}$ & & Het (1) & Splice_region_variant & 1 vs. 261 & 1366 vs. 125692 & $1 / 262=0.004$ & $1366 / 127058=0.01$ & $p=05376$ & $\begin{array}{c}\text { Not possible to } \\
\text { calculate }\end{array}$ \\
\hline \multirow{3}{*}{ DPP4 } & 1 & rs116302758 & $\begin{array}{l}\text { Chr2: } \\
162904013\end{array}$ & c. $95-2 A>G$ & & Het (6) & Splice_acceptor_variant & 6 vs. 256 & 4933 vs. 122045 & $6 / 262=0.023$ & $4933 / 126978=0.039$ & $p=0.2511$ & In equilibrium \\
\hline & 2 & rs56179129 & $\begin{array}{l}\text { Chr2: } \\
\text { 162890142 }\end{array}$ & c. $796 \mathrm{G}>\mathrm{A}$ & p.Val266lle & Het (2) & Missense_variant & 2 vs. 260 & 688 vs. 126666 & $2 / 262=0.008$ & $688 / 127354=0.005$ & $p=0.6557$ & In equilibrium \\
\hline & 3 & rs115450134 & $\begin{array}{c}\text { Chr2: } \\
162865748\end{array}$ & c. $1887+3 \mathrm{G}>\mathrm{A}$ & & Het (1) & Splice_region_variant & 1 vs. 261 & 1069 vs. 127981 & $1 / 262=0.004$ & $1069 / 129050=0.008$ & $p=0.7299$ & $\begin{array}{c}\text { Not possible to } \\
\text { calculate }\end{array}$ \\
\hline \multirow{5}{*}{ FURIN } & 1 & rs16944971 & $\begin{array}{l}\text { Chr15: } \\
\text { 91419098 }\end{array}$ & c. $128 \mathrm{C}>\mathrm{T}$ & p.Ala43Val & Het (1) & Missense_variant & 1 vs. 261 & 273 vs. 106605 & $1 / 262=0.004$ & $273 / 106878=0.0025$ & $p=0.4892$ & $\begin{array}{c}\text { Not possible to } \\
\text { calculate }\end{array}$ \\
\hline & 2 & rs780909157 & $\begin{array}{l}\text { Chr15: } \\
\text { 91419792 }\end{array}$ & c. $372+5 \mathrm{G}>\mathrm{A}$ & & Het (1) & Splice_region_variant & 1 vs. 261 & 6 vs. 24650 & $1 / 262=0.004$ & $6 / 24656=0.00024$ & $p=0.0713$ & $\begin{array}{l}\text { Not possible to } \\
\text { calculate }\end{array}$ \\
\hline & 3 & rs201551785 & $\begin{array}{l}\text { Chr15: } \\
\text { 91420189 }\end{array}$ & c. $436 \mathrm{G}>\mathrm{A}$ & p.Gly146Ser & Het (1) & Missense_variant & 1 vs. 261 & 51 vs. 129085 & $1 / 262=0.004$ & $51 / 129136=0.00039$ & $p=0.1$ & $\begin{array}{l}\text { Not possible to } \\
\text { calculate }\end{array}$ \\
\hline & 4 & rs769208985 & $\begin{array}{l}\text { Chr15: } \\
\text { 91422046 }\end{array}$ & c. $893 \mathrm{G}>\mathrm{A}$ & p.Arg298Gln & Het (1) & Missense_variant & 1 vs. 261 & 1 vs. 111907 & $1 / 262=0.004$ & $1 / 111908=0.0000089$ & $p=0.0047$ & $\begin{array}{l}\text { Not possible to } \\
\text { calculate }\end{array}$ \\
\hline & 5 & rs1236237792 & $\begin{array}{l}\text { Chr15: } \\
\text { 91424629 }\end{array}$ & c.1906A>G & p.Ile636Val & Het (1) & Missense_variant & 1 vs. 261 & Not reported & $1 / 262=0.004$ & Not reported & - & $\begin{array}{c}\text { Not possible to } \\
\text { calculate }\end{array}$ \\
\hline \multirow{5}{*}{ TMPRSS2 } & 1 & rs200291871 & $\begin{array}{l}\text { Chr21: } \\
42879910\end{array}$ & c. $22 \mathrm{G}>\mathrm{C}$ & p.Gly8Arg & Het (1) & Missense_variant & 1 vs. 261 & 360 vs. 32572 & $1 / 262=0.004$ & $360 / 32932=0.01$ & $p=0.5387$ & $\begin{array}{l}\text { Not possible to } \\
\text { calculate }\end{array}$ \\
\hline & 2 & rs75603675 & $\begin{array}{c}\text { Chr21: } \\
42879909\end{array}$ & c. $23 \mathrm{G}>\mathrm{T}$ & p.Gly8Val & $\begin{array}{l}\text { Het (55) } \\
\text { Hom (20) }\end{array}$ & Missense_variant & 95 vs. 157 & 14273 vs. 19331 & $95 / 262=0.36$ & $14273 / 33604=0.425$ & $p=0.0446$ & In equilibrium \\
\hline & 3 & rs61735791 & $\begin{array}{l}\text { Chr21: } \\
\text { 42866439 }\end{array}$ & c. $193 \mathrm{G}>\mathrm{A}$ & p.Ala65Thr & Het (2) & Missense_variant & 2 vs. 260 & 365 vs. 128739 & $2 / 260=0.008$ & $365 / 129104=0.0028$ & $p=0.1708$ & In equilibrium \\
\hline & 4 & rs114363287 & $\begin{array}{c}\text { Chr21: } \\
42866301 \\
\end{array}$ & c. $331 \mathrm{G}>\mathrm{A}$ & p.Gly111Arg & Het (1) & Missense_variant & 1 vs. 261 & 127779 & $1 / 262=0.004$ & $7 / 127786=0.000055$ & $p=0.0163$ & $\begin{array}{c}\text { Not possible to } \\
\text { calculate }\end{array}$ \\
\hline & 5 & rs12329760 & $\begin{array}{l}\text { Chr21: } \\
42852497\end{array}$ & c. $589 \mathrm{G}>\mathrm{A}$ & p.Val197Met & $\begin{array}{l}\text { Het (33) } \\
\text { Hom (6) }\end{array}$ & Missense_variant & 45 vs. 217 & 29831 vs. 98773 & $45 / 262=0.17$ & $29831 / 128604=0.23$ & $p=0.0228$ & In equilibrium \\
\hline
\end{tabular}


We also verified Hardy-Weinberg Equilibrium for those variants for which it was possible to calculate and the genotypes distributions resulted in HW equilibrium for all of them (Table 1).

To predict the potential impact on proteins of the rare identified variants (two in the PCSK3 gene and one in the TMPRSS2 gene), we used different tools (PolyPhen2, Mutation Taster, SIFT) [17-19], and two ensemble score (MetaLR_pred, MetaSVM_pred.). For the variant c.893G >A (p.Arg298Gln) identified in the PCSK3 gene, the in-silico analysis gave pathogenic computational verdict. The Arg residue at position 298 is a large hydrophilic amino acid, while the variant residue is a Gln, which has a neutral side chain. The sequence alignment of the furin protein with its orthologous proteins shows that the wild type residue is highly conserved in species ranging from human to zebrafish and lamprey (phastcons 46, in UCSC Genome browser) [20], implying an important functional or structural role for this residue in the furin protein. Also for the c.1906A $>\mathrm{G}$ (p.Ile636Val) in the PCSK3 gene, the wild type residue is highly conserved in species ranging from human to zebrafish and lamprey, but the change Ile in Val may not lead to changes in function as both amino acid residues have an aliphatic side chain and are hydrophobic. Indeed, in silico analysis gave benign computational verdict. Concerning the variants, c.331G > A (p.Gly111Arg) in the TMPRSS2 gene, the residue is conserved only in mammals and the in silico analysis gave benign computational verdict.

\section{Discussion}

We analyzed the genetic variants located on TMPSSR2, PCSK3, DPP4, and BSG coding-region in a representative cohort of Italian patients affected by COVID-19, in order to verify the hypothesis that the COVID-19 susceptibility is also influenced by genetic variability of genes coding for proteins involved in the entry of SARS-CoV-2 into target cells. By our preliminary study, we observed in the group of COVID-19 patients some differences in the frequencies of the genetic variants located in the two proteases studied, TMPRSS2 and PCSK3, compared to those reported in the GnomAD database.

Several studies have shown that SARS-CoV-2 entry is regulated by proteases. Proteolytic activation of $S$ protein potentially leads to the final structural change of $S 2$ needed for membrane fusion and the serine protease TMPRSS2 seems to be the main enzyme involved in this process [21]. In the COVID-19 patient group we identified the rare variant c.331G > A (p.Gly111Arg) localized in the TMPRSS2 gene, with a higher frequency compared to the GnomAD database.

A study conducted on an Italian cohort has shown that Italians had a significant decrease in the burden of deleterious variants in TMPRSS2 compared to the other Europeans populations, suggesting that they might have a higher level of TMPRSS2 protein or activity, which seems to represent a risk factor for a more severe disease course [22].

We also observed a lower frequency of c.23G $>$ T (p.Gly8Val) and c.589G $>$ A (p.Val197Met) polymorphic alleles in COVID-19 patients, compared to the allelic frequency of these variants in GnomAD for the EUR reference population. In particular, the missense variant c.589G $>A$ is located in the extracellular SRCR (Scavenger Receptor Cysteine-Rich) domain of TMPRSS2, which interacts with external pathogens [23]. The SRCR domain guides the protease domain, correctly orienting it towards its substrate and when absent, the proteolytic activity of TMPRSS2 decreases considerably, as demonstrated by functional studies. [24]. The minor variant allele of this polymorphism could alter the processing of SARS-CoV-2 S protein and thus present a protective effect towards infection.

Moreover, from bioinformatics analyses conducted by Zerubin et al. [24], TMPRSS2 was co-expressed with the main SARS-CoV-2 receptors (ACE2 and BSG). In addition, the authors extrapolated nine drugs from the DRUGBANK database that could reduce the expression levels of TMPRSS2. In particular, two of these drugs (Paracetamol and Curcumin) are currently being administered to alleviate some symptoms in COVID-19 patients [25]. In a study in vitro using cell lines and primary pulmonary cells, an inhibitor of the protease activity of TMPRSS2, camostat mesylate, partially inhibited the entry of SARS-CoV-2 into these lung epithelial cells [5]. These data suggest that inhibition of this protein may be a potential intervention to be investigated. 
TMPRSS2 was the first identified protease capable of cleaving the $S$ protein, but recent studies highlighted that SARS-CoV-2 contains a canonical furin-cleavage site, lacking in the other SARS-like CoVs, which facilitates its entry into the cells [7]. Furin is a member of the evolutionarily ancient family of proprotein convertases, called PCSK. Humans encode nine members of this protease family (PCSK1-9), with PCSK3 representing furin [26]. PCSK3 gene has low allelic frequencies of missense variants, as expected on the basis of GnomAD population data, but we have identified five of these rare variants in COVID-19 patients. In particular, the c.893G $>$ A causes an amino acid change from arginine to glycine in a very highly conserved position (R298) near the substrate-binding residues (292-295). In silico analysis showed that the variant does not alter the protein structure, but it could influence the recognition of the target sequence RxxR within SARS-CoV-2 spike protein.

Another interesting mutation identified in the PCSK3 gene is c.1906A $>\mathrm{G}$ (p.Ile636Val). This variant was predicted benign by in silico tools and it was not found in the GnomAD database. Therefore, its functional meaning should be clarified in subsequent studies.

PCSK3 is regulated by a family of proteins containing the EMI domain, a cysteine-rich sequence of approximately 80 aminoacids [27]. The EMILIN/Multimerin family includes EMILIN1, EMILIN2, Multimerin1, and Multimerin2: in the future it could be interesting to study also their possible genetic variants. Indeed, the EMI domain is characterized by seven cysteine residues located at regular positions and this domain is located at the $\mathrm{N}$-terminus in all the proteins in which it is expressed. EMILIN1, belonging to the EMILIN family, seems to bind upstream of the PCSK3 convertases and prevents the cleavage of its targets [28].

The choice of a patient group well clinically characterized and homogeneously and accurately diagnosed is an essential requirement in a genetic association study. In the case of COVID-19, the patients could have a high phenotypic variability, with different manifestations, ranging from very mild sub-clinical symptoms to ARDS. Therefore, it is difficult to define a homogeneous cohort, in which the patients under examination share symptoms and disease severity. Although the sample size appears sufficient to highlight differences in the genotypic frequency of the COVID-19 patient population compared to those reported in the GnomAD database, the number of subjects is too small to stratify them on the basis of clinical characteristics and clinical phenotypes. We consider this a limitation of our study. Besides, we consider that this preliminary study on the genetic variability of the genes involved in the entry of the virus could be useful for the scientific community. Moreover, an interesting data is that the three rare mutations whose frequencies were different with respect to the frequencies reported in the database, were all identified in the subgroup of "severe" patients.

Although genetic variants in these genes may influence entry of the SARS-CoV-2, currently there are no functional studies that can demonstrate their active role in susceptibility to infection. We can only hypothesize that mutations in these genes could alter protease functionality and facilitate the entry of the SARS-CoV-2, providing further confirmation of the importance of the spike protein activation process. From the data that we obtained, there are several differences in some of the genetic variants distribution with respect to Gnomad frequencies, but very strong associations with the disease do not seem to emerge. It would therefore be interesting to investigate in the future genetic variants in the intronic or regulatory regions of these genes, for example in the 5 'regions upstream of the promoters or in the 3' UTR sites, in the points of interaction with miRNAs. These preliminary results, although requiring further confirmation in larger independent cohorts and the population, as well as functional studies to evaluate the actual effect of the detected genetic variants, bring new supporting data to the role of human host genetic variability in the susceptibility to SARS-CoV-2 infection and its spread in human populations.

\section{Conclusions}

We believe that the variability in host susceptibility to infections, including SARS-CoV-2, is due to an interplay of different factors and our contribution in the dissection of the genetic ones could give 
support to these kind of studies, that could also be useful in identifying a higher-risk population that could also be prioritized for vaccination.

Author Contributions: A.L., P.B., M.B., A.N., and G.N. conceived the study; E.A., R.G., P.G., and M.H.-C. collected the data and performed the analyses. A.S., M.D., M.A., P.R., S.B., and G.N. contributed to the interpretation of the results and supervised the study. A.L., M.B., and P.B. wrote the manuscript; G.N., P.B., and M.B., revised the manuscript. A.L., E.A., A.N., P.B., R.G., P.G., A.S., M.D., P.R., and M.A. approved the final version of the manuscript. All authors have read and agreed to the published version of the manuscript.

Funding: This research was funded by Rotary Club Corigliano-Rossano "Sybaris", 2020.

Acknowledgments: This work is part of the GEFACOVID2.0 Consortium (http://www.lorenzinifoundation.org/ wp-content/uploads/2020/06/NEWS-GEFACOVID2.0_final-Consortium-News-GN-vEF.pdf). We are grateful to the Rotary Club Corigliano-Rossano "Sybaris" for the support and contribution to our research.

Conflicts of Interest: The authors declare no conflict of interest.

\section{References}

1. Sohrabi, C.; Alsafi, Z.; O’Neill, N.; Khan, M.; Kerwan, A.; Al-Jabir, A.; Iosifidis, C.; Agha, R. World Health Organization declares global emergency: A review of the 2019 novel coronavirus (COVID-19). Int. J. Surg. 2020, 76, 71-76. [CrossRef] [PubMed]

2. de Wit, E.; van Doremalen, N.; Falzarano, D.; Munster, V.J. SARS and MERS: Recent insights into emerging coronaviruses. Nat. Rev. Microbiol. 2016, 14, 523-534. [CrossRef] [PubMed]

3. Mingxuan, X.; Qiong, C. Insight into 2019 novel coronavirus-an updated interim review and lessons from SARS-CoV and MERS-CoV. Int. J. Infect. Dis. 2020, 94, 119-124. [CrossRef]

4. Letko, M.; Marzi, A.; Munster, V. Functional assessment of cell entry and receptor usage for SARS-CoV-2 and other lineage B betacoronaviruses. Nat. Microbiol. 2020, 5, 562-569. [CrossRef] [PubMed]

5. Shang, J.; Wan, Y.; Luo, C.; Ye, G.; Geng, Q.; Auerbach, A.; Li, F. Cell entry mechanisms of SARS-CoV-2. Proc. Natl. Acad. Sci. USA 2020, 117, 11727-11734. [CrossRef]

6. Hoffmann, M.; Kleine-Weber, H.; Schroeder, S.; Kruger, N.; Herrler, T.; Erichsen, S.; Schiergens, T.S.; Herrler, G.; $\mathrm{Wu}$, N.H.; Nitsche, A.; et al. SARS-CoV-2 cell entry depends on ACE2 and TMPRSS2 and is blocked by a clinically proven protease inhibitor. Cell 2020, 182, 271-280. [CrossRef]

7. Coutard, B.; Valle, C.; de Lamballerie, X.; Canard, B.; Seidah, N.G.; Decroly, E. The spike glycoprotein of the new coronavirus 2019-nCoV contains a furin-like cleavage site absent in $\mathrm{CoV}$ of the same clade. Antivir. Res. 2020, 176, 104742. [CrossRef]

8. Millet, J.K.; Whittaker, G.R. Host cell proteases: Critical determinants of coronavirus tropism and pathogenesis. Virus Res. 2015, 202, 120-134. [CrossRef]

9. Vankadari, N.; Wilce, J.A. Emerging COVID-19 coronavirus: Glycan shield and structure prediction of spike glycoprotein and its interaction with human CD26. Emerg. Microbes Infect. 2020, 9, 601-604. [CrossRef]

10. Wang, K.; Chen, W.; Zhou, Y.S.; Lian, J.Q.; Zhang, Z.; Du, P.; Gong, L.; Zhang, Y.; Cui, H.Y.; Geng, J.J.; et al. SARS-CoV-2 invades host cells via a novel route: CD147-spike protein. BioRxiv 2020. [CrossRef]

11. Ulrich, H.; Pillat, M.M. CD147 as a target for COVID-19 treatment: Suggested effects of azithromycin and stem cell engagement. Stem Cell Rev. Rep. 2020, 16, 434-440. [CrossRef] [PubMed]

12. Online Mendelian Inheritance in Man, OMIM. Available online: https://OMIM.org/ (accessed on 10 July 2020).

13. Novelli, A.; Biancolella, M.; Borgiani, P.; Cocciadiferro, D.; Colona, V.L.; D’Apice, M.R.; Rogliani, P.; Zaffina, S.; Leonardis, F.; Campana, A.; et al. Analysis of ACE2 Genetic Variants in 131 Italian SARS-CoV-2 Positive Patients. 2020. Available online: https://www.researchsquare.com/article/rs-39011/v3 (accessed on 26 August 2020). [CrossRef]

14. Verdoni, L.; Mazza, A.; Gervasoni, A.; Martelli, L.; Ruggeri, M.; Ciuffreda, M.; Bonanomi, E.; D’Antiga, L. An outbreak of severe Kawasaki-like disease at the Italian epicentre of the SARS-CoV-2 epidemic: An observational cohort study. Lancet 2020, 395, 1771-1778. [CrossRef]

15. GnomAD. Available online: https://gnomad.broadinstitute.org/ (accessed on 10 July 2020).

16. Karczewski, K.J.; Francioli, L.C.; Tiao, G.; Cummings, B.B.; Alföldi, J.; Wang, Q.; Collins, R.L.; Laricchia, K.M.; Ganna, A.; Birnbaum, D.; et al. The mutational constraint spectrum quantified from variation in 141,456 humans. Nature 2020, 581, 434-443. [CrossRef] [PubMed]

17. PolyPhen2. Available online: http://genetics.bwh.harvard.edu/pph2/ (accessed on 10 July 2020). 
18. Mutation Taster. Available online: http://www.mutationtaster.org/ (accessed on 10 July 2020).

19. Sorting Intolerant from Tolerant, SIFT. Available online: https://sift.bii.a-star.edu.sg/ (accessed on 10 July 2020).

20. UCSC Genome Browser. Available online: https://genome.ucsc.edu (accessed on 10 July 2020).

21. Tang, T.; Bidon, M.; Jaimes, J.A.; Whittaker, G.R.; Daniel, S. Coronavirus membrane fusion mechanism offers a potential target for antiviral development. Antivir. Res. 2020, 178, 104792. [CrossRef]

22. Asselta, R.; Paraboschi, E.M.; Mantovani, A.; Duga, S. ACE2 and TMPRSS2 variants and expression as candidates to sex and country differences in COVID-19 severity in Italy. Aging 2020, 12, 10087-10098. [CrossRef]

23. Somoza, J.R.; Ho, J.D.; Luong, C.; Ghate, M.; Sprengeler, P.A.; Mortara, K.; Shrader, W.D.; Sperandio, D.; Chan, H.; McGrath, M.E.; et al. The structure of the extracellular region of human hepsin reveals a serine protease domain and a novel scavenger receptor cysteine-rich (SRCR) domain. Structure 2003, 11, 1123-1131. [CrossRef]

24. Böttcher-Friebertshäuser, E.; Freuer, C.; Sielaff, F.; Schmidt, S.; Eickmann, M.; Uhlendorff, J.; Steinmetzer, T.; Klenk, H.D.; Garten, W. Cleavage of influenza virus hemagglutinin by airway proteases TMPRSS2 and HAT differs in subcellular localization and susceptibility to protease inhibitors. J. Virol. 2010, 84, 5605-5614. [CrossRef]

25. Zarubin, A.; Stepanov, V.; Markov, A.; Kolesnikov, N.; Marusin, A.; Khitrinskaya, I.; Swarovskaya, M.; Litvinov, S.; Ekomasova, N.; Dzhaubermezov, M.; et al. Structural variability, expression profile and pharmacogenetics properties of TMPRSS2 gene as a potential target for COVID-19 therapy. BioRxiv 2020. [CrossRef]

26. Braun, E.; Sauter, D. Furin-mediated protein processing in infectious diseases and cancer. Clin. Transl. Immunol. 2019, 8, 1073. [CrossRef]

27. Zacchigna, L.; Vecchione, C.; Notte, A.; Cordenonsi, M.; Dupont, S.; Maretto, S.; Cifelli, G.; Ferrari, A.; Maffei, A.; Fabbro, C.; et al. Emilin1 links TGF-beta maturation to blood pressure homeostasis. Cell 2006, 124, 929-942. [CrossRef]

28. Colombatti, A.; Spessotto, P.; Doliana, R.; Mongiat, M.; Bressan, G.M.; Esposito, G. The EMILIN/Multimerin family. Front. Immunol. 2012, 2, 93. [CrossRef] [PubMed]

(C) 2020 by the authors. Licensee MDPI, Basel, Switzerland. This article is an open access article distributed under the terms and conditions of the Creative Commons Attribution (CC BY) license (http://creativecommons.org/licenses/by/4.0/). 